\title{
Relating quantum privacy and quantum coherence: an operational approach
}

\author{
I. Devetak ${ }^{1}$ and A. Winter ${ }^{2}$ \\ ${ }^{1}$ IBM T. J. Watson Research Center, PO Box 218, Yorktown Heights, NY 10598, USA \\ ${ }^{2}$ Department of Computer Science, University of Bristol, Bristol BS8 1UB, U.K.
}

(Dated: July 8, 2003)

\begin{abstract}
We describe how to achieve optimal entanglement generation and one-way entanglement distillation rates by coherent implementation of a class of secret key generation and secret key distillation protocols, respectively.
\end{abstract}

Quantum information theory may be understood in terms of inter-conversion between various resources [9]. One very desirable resource is a maximally entangled state shared between two parties (Alice and Bob). It allows them to perform various tasks such as teleportation, super-dense coding, etc. [5]. Another is a pair of maximally correlated classical bit-strings shared by the two parties, reliably secret from any third party (Eve). It has important cryptographic applications since it can be used as a secret key (or "one time pad") allowing Alice and Bob private communication over a public channel. Although used for different purposes, the two resources are intimately related through the property of "exclusiveness" - in the one case toward the total outside world, in the other toward the eavesdropper Eve. This connection has been confirmed by a growing literature of useful analogies [7, 19] and operational equivalences $[1,21]$. In this Letter we further exploit this connection to relate several private and quantum protocols, culminating in the demonstration of the "hashing inequality" for one-way entanglement distillation [6, 13]. We consider four distinct resource conversion scenarios, starting from a noisy quantum channel/entanglement and ending up with near-perfect secret key/entanglement. In other words we are converting a noisy dynamic/static quantum resource into a noiseless static private/quantum resource.

1. static $\rightarrow$ private $=$ Secret key distillation. The task is to convert $n$ copies of the bipartite state $\rho^{A B}$ shared between Alice and Bob into $n R$ bits of secret key using 1-LOPC (local quantum operations and forward public communication). The quantity $R$ is referred to as the rate of the protocol. To understand what is meant by a secret key, consider a purification $\psi^{A B E}=|\psi\rangle\left\langle\left.\psi\right|^{A B E}\right.$ of $\rho^{A B}$ and allow for the worst case scenario in which the eavesdropper Eve is given the purifying system $E$. Defining $\bar{\Phi}=\frac{1}{2}(|00\rangle\langle 00|+| 11\rangle\langle 11|)$, a pair of maximally correlated bits written formally as a density operator, the desired shared secret key is represented as $\left(\bar{\Phi}^{A B}\right)^{\otimes n R} \otimes$ $\theta^{E}$; the classical key shared between Alice and Bob is decoupled from Eve's state $\theta$.

2. static $\rightarrow$ quantum $=$ Entanglement distillation. Here, by 1-LOCC (local quantum operations and forward clas- sical communication) $\left(\rho^{A B}\right)^{\otimes n}$ is to be converted into $n R$ bits of entanglement $\Phi_{+}^{\otimes n R}$, where $\left|\Phi_{+}\right\rangle=\frac{1}{\sqrt{2}}(|00\rangle+|11\rangle)$. Since the final state is pure, the decoupling from $E$ is implicit. Including $E$ in the description, the effect of the protocol may be written as $\left(\psi^{A B E}\right)^{\otimes n} \Longrightarrow\left(\Phi_{+}^{A B}\right)^{\otimes n R} \otimes$ $\theta^{E}$, which is just a coherent version of its private counterpart above!

3. dynamic $\rightarrow$ private $=$ Secret key generation. Here the starting point is a noisy quantum channel $\mathcal{N}$, a cptp map taking density operators in the Hilbert space of Alice's system $A^{\prime}$ to that of Bob's $B$. Applying $\mathcal{N}$ is equivalent to an isometric mapping onto a larger Hilbert space corresponding to $B E$, which includes the environment $E$ assumed at Eve's disposal. The channel may be characterized by its effect on some bipartite pure state $\left|\psi^{\prime}\right\rangle^{A A^{\prime}}$, living entirely on Alice's side, which now becomes the mixed state $\rho^{A B}=\left(\mathbb{1}^{A} \otimes \mathcal{N}\right) \psi^{\prime}$ shared between her and Bob. Note that the purifying system for $\rho^{A B}$ is precisely $E$, so that we again have the tripartite state $\psi^{A B E}$. The task is, by Alice choosing an appropriate input to $\mathcal{N}^{\otimes n}$ and Bob performing a decoding operation, to generate $n R$ bits of secret key, decoupled from $E$ as in the first scenario.

4. dynamic $\rightarrow$ quantum $=$ Entanglement generation . The channel $\mathcal{N}$ is now used to generate $n R$ bits of entanglement. Again one may explicitly include $E$ to stress the resemblance to the private scenario.

We shall show how a particular asymptotic rate $R$ defined in terms of the state $\psi^{A B E}$ may be achieved for the four scenarios, by which we mean that for sufficiently large $n$ there exists a protocol whose output approximates the desired state arbitrarily closely (in trace distance). First we give a simple dimension counting argument for secret key generation, and show how to augment it to work for secret key distillation. Then we modify the two protocols to make them "coherent", yielding the entanglement generation and distillation protocols, respectively. Finally, we point to a more direct connection between the two coherent protocols and discuss the implications of our results for finding optimal rates. A rigorous treatment of the dynamic and static scenarios via random code selection may be found in [8] and [10], respectively, building on results from classical information theory [2].

The state $\psi^{A B E}$, related to our coherent protocols, may be written in the Schmidt representation with re- 
spect to the $A \mid B E$ partition as

$$
|\psi\rangle^{A B E}=\sum_{x} \sqrt{P(x)}|x\rangle^{A} \otimes\left|\phi_{x}\right\rangle^{B E} .
$$

In the dynamic scenario it comes about by sending some $|\psi\rangle^{A A^{\prime}}=\sum_{x} \sqrt{P(x)}|x\rangle^{A} \otimes\left|\phi_{x}^{\prime}\right\rangle^{A^{\prime}}$ through the channel. Relevant to our private protocols is the "decohered" state

$$
\bar{\psi}^{A B E}=\sum_{x} P(x)|x\rangle\left\langle\left. x\right|^{A} \otimes \phi_{x}^{B E},\right.
$$

obtainable from $|\psi\rangle^{A B E}$ by measurement in the $\{|x\rangle\}$ basis. It represents the state of a cqq system XBE [9], $X$ being a random variable with probability distribution $P(x)$. In the dynamic scenario it arises from sending the ensemble $\left\{P(x),\left|\phi_{x}^{\prime}\right\rangle^{A^{\prime}}\right\}$ through the channel.

Define $\omega=\bar{\psi}^{B}=\psi^{B}$ and $\sigma=\bar{\psi}^{E}=\psi^{E}$. For a quantum state $\rho$ we denote the von Neumann entropy $H(\rho)=-\operatorname{Tr} \rho \log \rho$, and the Shannon entropy of a random variable $X, H(X)=-\sum_{x} P(x) \log P(x)$. If the state is the reduced state of a multi-party state, like the $\bar{\psi}^{A B E}$ above, we write $H(A)=H\left(\bar{\psi}^{A}\right)$, etc. In the particular case of (1), obviously $H\left(\bar{\psi}^{A}\right)=H(X)$. For a general bipartite state on $A B$ define the conditional entropy $H(A \mid B)=H(A B)-H(B)$ and quantum mutual information

$$
I(A ; B)=H(A)+H(B)-H(A B) .
$$

The latter for the cqq state in (1) is easily checked to be equal to the Holevo information $H(\omega)-\sum_{x} P(x) H\left(\phi_{x}^{B}\right)$ 11] for which we shall use the notation $I(X ; B)$. As a rule, information theoretical quantities involving $X$ are implicitly referring to $\bar{\psi}^{A B E}$, and those involving $A$ to $\psi^{A B E}$. For instance, the coherent information $\left.I_{c}(A\rangle B\right)=-H(A \mid B)$ [18] refers to $\psi^{A B E}$, but may be written as $H(B)-H(E)=I(X ; B)-I(X ; E)$ when referring to $\bar{\psi}^{A B E}$. The protocols we are about to describe will all achieve the rate $\left.R=I_{c}(A\rangle B\right)$. In the entanglement distillation scenario this is known as the "hashing inequality" 6, 13].

Typicality. Let us review the properties of typical sequences and subspaces. For the random variable $X$, any $\epsilon, \delta>0$ and sufficiently large $n$ there exists a typical set $T_{X, \delta}^{n}$ consisting of sequences $x^{n}=x_{1} x_{2} \ldots x_{n}$ of length $n$ such that

$$
2^{n[H(X)-\delta]} \leq\left|T_{X, \delta}^{n}\right| \leq 2^{n[H(X)+\delta]},
$$

and $\operatorname{Pr}\left\{X^{n} \in T_{X, \delta}^{n}\right\} \geq 1-\epsilon$. Typical sequences are those in which the fraction of a given letter $x$ is approximated by its probability $P(x)$, and the law of large numbers guarantees that such sequences will occur with high probability. In less formal notation, large $n$ will be implicit, the typical set denoted by $T_{X}, H(X) \pm \delta$ written as $H(X)^{ \pm}$and $1-\epsilon$ as $\approx 1$.
The quantum analogue of the typical set is the typical subspace 17] $\mathcal{T}_{B}$ of the Hilbert space $\mathcal{H}_{B}^{\otimes n}$, defined for the quantum system $B$ in the state $\omega$. It satisfies

$$
2^{n H(B)^{-}} \leq \operatorname{dim} \mathcal{T}_{B} \leq 2^{n H(B)^{+}},
$$

and $\omega^{\otimes n}$ is approximately supported on $\mathcal{T}_{B}$ in the sense that $\Pi_{B} \omega^{\otimes n} \Pi_{B} \approx \omega^{\otimes n}$ (in trace distance) with $\Pi_{B}$ the projector onto $\mathcal{T}_{B}$.

For a cq system $X B$ and a particular sequence $x^{n} \in T_{X}$ there exists a conditionally typical subspace $\mathcal{T}_{B \mid X}\left(x^{n}\right)$ on which $\phi_{x^{n}}^{B}=\bigotimes_{i} \phi_{x_{i}}^{B}$ is approximately supported, such that

$$
2^{n H(B \mid X)^{-}} \leq \operatorname{dim} \mathcal{T}_{B \mid X}\left(x^{n}\right) \leq 2^{n H(B \mid X)^{+}} .
$$

Another important fact is that the $\mathcal{T}_{B \mid X}\left(x^{n}\right)$ can be thought of as being approximately contained in $\mathcal{T}_{B}$ in the sense that $\Pi_{B} \Pi_{B \mid X}\left(x^{n}\right) \Pi_{B}$ enjoys the same asymptotic properties as $\Pi_{B \mid X}\left(x^{n}\right)$.

$H S W$ codes. An HSW 12 code $\mathcal{C}$ associated with the Alice-Bob cq system $X B$ is a subset of $T_{X}$ such that the states $\left(\psi_{x^{n}}^{B}\right)_{x^{n} \in \mathcal{C}}$ can be distinguished with probability $\approx 1$. Intuitively, the $\psi_{x^{n}}^{B}$, supported on the respective $\mathcal{T}_{B \mid X}\left(x^{n}\right)$ of dimension at most $2^{n H(B \mid X)^{+}}$, can be "packed" into $\mathcal{T}_{B}$ of dimension at least $2^{n H(B)^{-}}$with negligible overlap if we take $|\mathcal{C}|=2^{n I(X ; B)^{-}}$(Fig. 1). The HSW theorem [12] confirms this geometric picture.

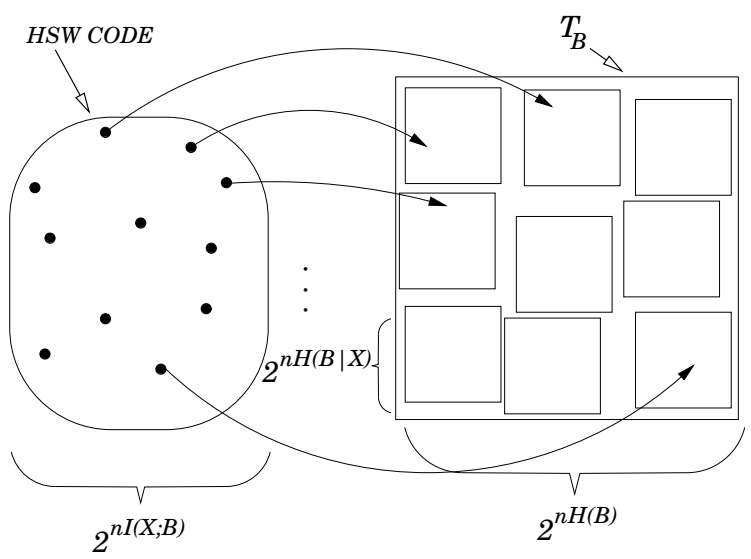

FIG. 1: An HSW code. The arrows represent the map $x^{n} \mapsto$ $\phi_{x^{n}}^{B}$.

Privacy amplification sets. Privacy amplification (PA) sets are, in a sense, dual to HSW codes. A privacy amplification set $\mathcal{S}$, associated with the Alice-Eve cq system $X E$, is a subset of $T_{X}$ of size $S$ such that

$$
\frac{1}{S} \sum_{x^{n} \in \mathcal{S}} \phi_{x^{n}}^{E} \approx \sigma^{\otimes n} .
$$

Evidently $\mathcal{S}=T_{X}$ is a valid PA set, but we are interested in making $S$ as small as possible. Since $\sigma^{\otimes n}$ and $\phi_{x^{n}}^{E}$ are "almost" uniformly supported on $\mathcal{T}_{E}$ and $\mathcal{T}_{E \mid X}\left(x^{n}\right)$, respectively, satisfying (2) amounts to "covering" $\mathcal{T}_{E}$, of 
dimension at most $2^{n H(E)^{+}}$with the $\mathcal{T}_{E \mid X}\left(x^{n}\right), x^{n} \in \mathcal{S}$, each of which has dimension at least $2^{n H(E \mid X)^{-}}$(Fig. 2 ). It can be shown (using techniques from [3]) that the quotient of these dimensions, $S=2^{n I(X ; E)^{+}}$, indeed suffices.

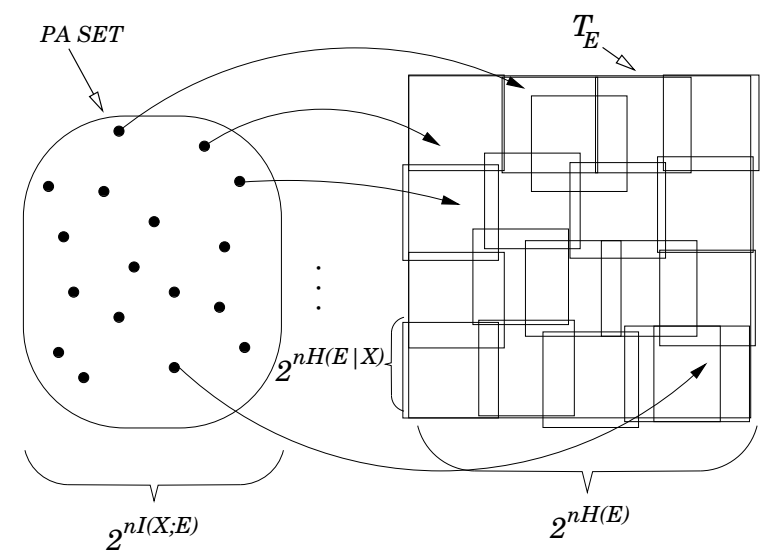

FIG. 2: A privacy amplification set. The arrows represent the map $x^{n} \mapsto \phi_{x^{n}}^{E}$.

Key generation (3.). We have seen that randomizing over the members of the PA set leaves Eve with a state essentially independent of the choice of $\mathcal{S}$. The key generation strategy is now clear. Construct an HSW code $\left(u^{m s}\right)_{m, s}, m=1, \ldots M, M=2^{n R^{-}}, s=1, \ldots S$, of size $M S=2^{n I(X ; B)^{-}}$such that each $\mathcal{S}_{m}=\left(u^{m s}\right)_{s}$ is a PA set. The key generation code is such a partitioned HSW code (Fig. 3). Alice sends the ensemble $\left\{\frac{1}{M}, \frac{1}{S} \sum_{s} \phi^{\prime}{ }_{u^{m s}}\right\}$ through the channel, resulting in the cq state

$$
\bar{\Psi}^{A B E}=\frac{1}{M} \sum_{m}|m\rangle\left\langle\left. m\right|^{A} \otimes \frac{1}{S} \sum_{s} \phi_{u^{m s}}^{B E} .\right.
$$

Bob measures his system to find out $m$ and Eve is left with the state $\theta^{E} \approx \sigma^{\otimes n}$ independent of the index $m$.

Key distillation (1.). The geometric idea (Fig. 3) is to cover the space $T_{X}$ of dimension at most $2^{n H(X)^{+}}$with $L=2^{n H(X \mid B)^{+}}$key generation codes $\mathcal{C}_{l}=\left(u^{l m s}\right)_{m, s}$ of size at least $2^{n I(X ; B)^{-}}$. Alice converts $\psi^{A B E}$ into $\bar{\psi}^{A B E}$ for all $n$ copies by measurement in the $\{|x\rangle\}$ basis. The measurement outcome $x^{n}$ with probability $\approx 1$ lies in $T_{X}$. Due to the covering, $x^{n}$ lies in some key generation code labeled by $l$. Alice sends the "which key generation code" information $l$ (this requires $n I(X \mid B)^{+}$bits), leaves the overall system in the state $\bar{\Psi}^{A B E}[16]$ and Bob simply proceeds as in key generation. The extra classical communication thus compensates for the initial resource being static rather than dynamic.

We now show how the two private protocols can be made coherent.

Entanglement generation (4.). For entanglement generation Alice prepares $\frac{1}{\sqrt{M}} \sum_{m}|m\rangle^{A} \otimes\left|\varphi_{m}\right\rangle^{A^{\prime}}$, the quan-

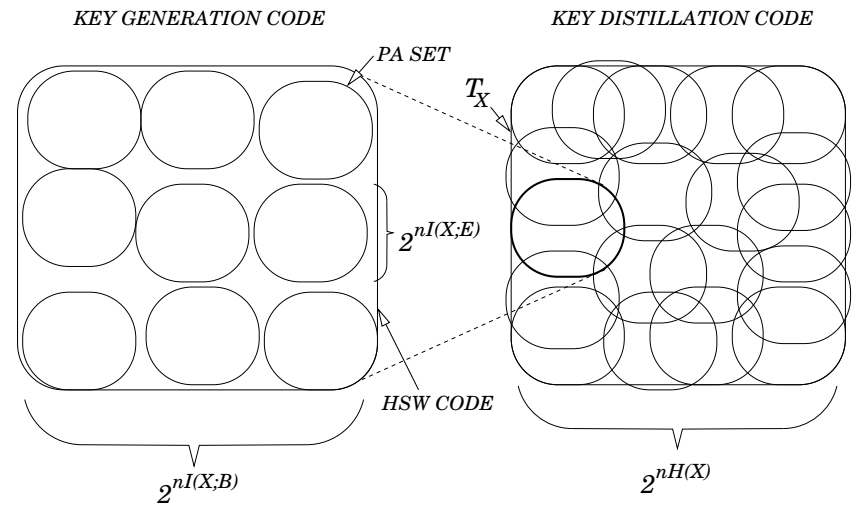

FIG. 3: A secret key generation code (left) viewed as a building block for a key distillation code (right).

tum code $\left(\left|\varphi_{m}\right\rangle\right)_{m}$ being defined by

$$
\left|\varphi_{m}\right\rangle=\frac{1}{\sqrt{S}} \sum_{s}\left|\phi_{u^{m s}}^{\prime}\right\rangle .
$$

Upon applying the channel this becomes

$$
|\Psi\rangle^{A B E}=\frac{1}{\sqrt{M}} \sum_{m}|m\rangle^{A} \otimes \frac{1}{\sqrt{S}} \sum_{s}\left|\phi_{u^{m s}}\right\rangle^{B E} .
$$

The remainder of the protocol consists of Bob decoding operation. He performs the measurement of $m s$ coherently by appending a register $B^{\prime}$ in a standard state $|0\rangle$, performing a unitary operation that places the measurement "outcome" $m s$ into $B^{\prime}$ and swapping the contents of $B$ and $B^{\prime}$. Since the original measurement yielded the correct value of $m s$ with probability $\approx 1$, the coherent measurement can be constructed to output an approximation of

$$
\frac{1}{\sqrt{M S}} \sum_{m s}|m\rangle^{A} \otimes|m s\rangle^{B}\left|\phi_{u^{m s}}\right\rangle^{B^{\prime} E} .
$$

This can be rewritten as

$$
\begin{aligned}
& \frac{1}{\sqrt{M}} \sum_{m}|m\rangle^{A} \otimes|m\rangle^{B}\left|\widetilde{\phi}_{m}\right\rangle^{B^{\prime} E} \text {, where } \\
& \left|\widetilde{\phi}_{m}\right\rangle^{B^{\prime} E}=\frac{1}{\sqrt{S}} \sum_{s}|s\rangle^{B_{1}^{\prime}}\left|\phi_{u^{m s}}\right\rangle^{B_{2}^{\prime} E} .
\end{aligned}
$$

Observe that $\widetilde{\phi}_{m}^{E} \approx \theta^{E}$; by Uhlmann's theorem their purifications are related as $\left|\widetilde{\phi}_{m}\right\rangle \approx\left(V_{m} \otimes \mathbb{1}\right)\left|\phi_{\theta}\right\rangle$ for some unitary operator $V_{m}$. Performing the controlled unitary $V=\sum_{m}|m\rangle\langle m| \otimes V_{m}$ on the system $B B^{\prime}$ finally decouples Eve, yielding

$$
\frac{1}{\sqrt{M}} \sum_{m}|m\rangle^{A} \otimes|m\rangle^{B}\left|\phi_{\theta}\right\rangle^{B^{\prime} E} .
$$

The above protocol may be modified into an entanglement transmission one 4], in which case Alice encodes a quantum state via $\sum_{m}\left|\varphi_{m}\right\rangle\langle m|$. Hence our use of the term "quantum code" for $\left(\left|\varphi_{m}\right\rangle\right)_{m}$. 
Entanglement distillation (2.). We now turn to the construction of a coherent version of the key distillation protocol. To each classical set $\mathcal{C}_{l}$ corresponds a quantum operator $\Lambda_{l}=\sum_{m s}|m s\rangle\left\langle u^{l m s}\right|$. In lieu of a complete von Neumann measurement on $A$, Alice performs a much less intrusive one composed of the $\Lambda_{l}$ (there is also a "failure" outcome that happens with probability $\approx 0$ ), revealing a particular value of $l$ which is communicated to Bob using $n H(X \mid B)^{+}$bits. Their joint state becomes [16]

$$
|\Psi\rangle^{A B E}=\frac{1}{\sqrt{M}} \sum_{m}|m s\rangle^{A} \otimes \frac{1}{\sqrt{S}} \sum_{s}\left|\phi_{u^{l m s}}\right\rangle^{B E} .
$$

which differs from (4) only in that $|m\rangle^{A}$ is replaced by $|m s\rangle^{A}$. As before, Bob performs the coherent measurement, resulting in the analogue of (5):

$$
\frac{1}{\sqrt{M S}} \sum_{m s}|m s\rangle^{A} \otimes|m s\rangle^{B}\left|\phi_{u^{l m s}}\right\rangle^{B^{\prime} E} \text {. }
$$

To dispose of Alice's s-register in a coherent way further classical communication is necessary. Alice performs a measurement in the Fourier-transformed basis $|\hat{t}\rangle=\frac{1}{\sqrt{S}} \sum_{s=1}^{S} e^{2 \pi i s t / S}|s\rangle(t=1, \ldots, S)$, and communicates the result $t$ to Bob using $n I(X ; E)^{+}$bits, who then applies the phase shift $\sum_{s=1}^{S} e^{2 \pi i s t / S}|s\rangle\langle s|$ to the $s^{-}$ component of his $B$ register. This yields precisely (5) and the rest of the protocol follows the entanglement generation one above.

It is possible to make a more direct connection between entanglement distillation and the quantum codes used for entanglement generation. Observe that the quantum code (3) could have equally well been substituted by $\left(\left|\phi_{l t m}\right\rangle\right)_{m}$ for any $t=1, \ldots, S, l=1, \ldots, L$, where

$$
\left|\phi_{l t m}\right\rangle=\frac{1}{\sqrt{S}} \sum_{s} e^{2 \pi i s t / S}\left|\phi_{u^{l m s}}\right\rangle .
$$

The sets $\left(\left|\phi_{l t m}\right\rangle\right)_{t m}$ and $\left(\left|\phi_{u^{l m s}}\right\rangle\right)_{m s}$ are (mutually unbiased) bases for the same space $\mathcal{Q}_{l}$. The $\mathcal{Q}_{l}$ are, in turn, a covering of $W^{\otimes n}\left(\mathcal{T}_{A}\right), W=\sum_{x}\left|\phi_{x}\right\rangle\langle x|$, since the $\mathcal{C}_{l}$ are a covering of $T_{X}$. In other words, $W^{\otimes n}\left(\mathcal{T}_{A}\right)$ is covered by quantum codes in much the same way that $T_{X}$ is covered by key generation codes. Our entanglement distillation protocol may be viewed as Alice collapsing her space onto some quantum code via a measurement, sending $I(A ; E)^{+}$bits of "which code" information to Bob, and Bob decoding as in entanglement generation. This view is very similar to the approach pursued by the Horodeckis [14], the difference lying in our using "random CSS codes" 8] rather than random subspace ones [15, 20].

In conclusion, we have seen that all four scenarios allow an asymptotic conversion rate $\left.R=I_{c}(A\rangle B\right)$, the latter referring to the state $\rho^{A B}$ which is either given (static case), or can be created by an application of the channel (dynamic case). In fact, the optimal rates for the coherent scenarios are also given in terms of $I_{c}$, but applied to an appropriately blocked and preprocessed state/channel 8, 10, 13]. The same is true for the private scenarios with $\left.I_{c}(A\rangle B\right)$ replaced by the possibly larger $I(X ; A)-I(X ; B)[22]$. It remains an open question whether coherent (quantum) and private information are fully equivalent within our model.

We thank A. Harrow, D. Leung and P. Hayden for useful comments and discussions. ID is supported by the NSA under the ARO grant numbers DAAG55-98-C-0041 and DAAD19-01-1-06. AW is supported by the U.K. Engineering and Physical Sciences Research Council.

[1] A. Acín, L. Masanes, N. Gisin, quant-ph/0303053 2003; D. Bruß, M. Christandl, A. Ekert, B.-G. Englert, D. Kaszlikowski, C. Macchiavello, quant-ph/0303184 2003.

[2] R. Ahlswede, I. Csiszár, IEEE Trans. Inf. Theory 39(4):1121, 1993.

[3] R. Ahlswede, A. Winter, IEEE Trans. Inf. Theory 48(3):569, 2002.

[4] H. Barnum, M. Knill, M. A. Nielsen, IEEE Trans. Inf. Theory 46(4):1317, 2000.

[5] C. H. Bennett, S. Wiesner, Phys. Rev. Lett. 69(20):2881, 1992; C. H. Bennett, G. Brassard, C. Crépeau, R. Jozsa, A. Peres, W. K. Wootters, Phys. Rev. Lett. 70(13):1895, 1993.

[6] C. H. Bennett, D. P. DiVincenzo, J. A. Smolin, W. K. Wootters, Phys. Rev. A 54(5):3824, 1996.

[7] D. Collins, S. Popescu, Phys. Rev. A 65(3):032321, 2002.

[8] I. Devetak, quant-ph/0304127. 2003; N. Cai, R. Yeung, "Quantum Privacy and Quantum Wiretap Channels", in preparation.

[9] I. Devetak, A. Winter, quant-ph/0304196 2003.

[10] I. Devetak, A. Winter, quant-ph/0306078 2003.

[11] A. S. Holevo, Probl. Pered. Inf. 9(3):3, 1973.

[12] A. S. Holevo, IEEE Trans. Inf. Theory 44(1):269, 1998; B. Schumacher, M. D. Westmoreland, Phys. Rev. A 56(1):131, 1997.

[13] M. Horodecki, P. Horodecki, R. Horodecki, Phys. Rev. Lett. 85(2):433, 2000.

[14] M. Horodecki, P. Horodecki, "Hashing Inequality", in preparation.

[15] S. Lloyd, Phys. Rev. A 55(3):1613, 1997.

[16] In fact, the distribution over the $m$ is not exactly even as in the dynamic case and the obtained secret key (entanglement) needs to be further "concentrated". In [10] this issue is avoided by measuring the "type" of $X(A)$ from the start.

[17] B. Schumacher, Phys. Rev. A 51(4):2738, 1995.

[18] B. Schumacher, Phys. Rev. A 54(4):2614, 1996; B. Schumacher, M. A. Nielsen, Phys. Rev. A 54(4):2629, 1996.

[19] B. Schumacher, M. D. Westmoreland, Phys. Rev. Lett. 80(25):5695, 1998.

[20] P. W. Shor, "The quantum channel capacity and coherent information", in preparation.

[21] P. W. Shor, J. Preskill, Phys. Rev. Lett. 85(2):441, 2000.

[22] The $\phi_{x}^{\prime}$ may be taken to be mixed if coherence is not required, corresponding to non-maximal measurements in key distillation. 